\title{
On Making a Difference: Towards an Integrative Framework for Green IT and Green IS Adoption
}

\author{
Helge Schmermbeck \\ University of Duisburg-Essen \\ helge.schmermbeck@uni-due.de
}

\begin{abstract}
Organizations and society nowadays face significant challenges. Organizations are required to fundamentally digital transform by assimilating Information Technology (IT) and Information System (IS) assets. Society faces an increasingly severe global climate disruption and needs to become more environmentally friendly. Green IT (GIT) and Green IS (GIS), as technologies and initiatives that seek to reduce the negative impacts of IT/IS on the environment, are a response to this. They can help organizations to gain a competitive advantage while also addressing broadscale environmental issues. We undertake a literature review to frame the general GIT/GIS adoption process. We provide an overarching understanding by modeling a sequence of five cognitive adoption phases (outset, pre-adoption, adoption, post-adoption, and outcome) on four levels (environmental, societal, organizational, and individual). By recognizing that GIT/GIS adoption has multiple drivers and outcomes, we provide an extensive perspective on GIT/GIS adoption.
\end{abstract}

\section{Introduction}

Green denotes artifacts that "positively impact the environment" [1], and, in the Information Systems (IS) domain, are primarily linked to Green Information Technologies (GIT). Although GIT has been defined in various ways, it originally described "technologies and initiatives to reduce the power, cooling and real estate costs associated with data center operations" [2]. This understanding served as the foundation of the Green IS (GIS) concept, which refers to utilizing Information Technology (IT) and IS for making organizations more sustainable and green [3]. Overall, GIS is extensive to GIT, since it also focuses on business processes and the sociotechnical interplays of persons and IT [4].

Concerning the different capitalizations of GIT and GIS [5] and in the ongoing discourse about using them distinctively [6], interchangeability [7], and integratively [8], we agree with the latter, viewing GIT as "measures and initiatives which decrease the negative environmental impact of manufacturing, operations, and disposal of Information Technology (IT) equipment and infrastructure" [8], and GIS as "practices which determine the investment in, deployment, use and management of Information Systems (IS) in order to minimize the negative environmental impact of IS, business operations, and IS-enabled products and services" [8]. To address both GIT and GIS, we use GIT/GIS.

Fundamentally, GIT/GIS are innovations that any adopting entity (AE) (e.g. organizations, individuals) evaluates in a cognitive innovation decision process (gain initial knowledge of, form an attitude towards, and make a decision to adopt or reject the innovation [9]) and thus may choose to adopt, or not [10]. While an organization's adoption of a novelty is traditionally understood as a quest for greater economic benefit, we acknowledge that the adoption of green technologies differs [11]. GIT/GIS adoption seems only secondarily driven by economic intentions (e.g. lower costs, improved systems performance) but is driven by ethical and sustainable considerations (e.g. reduce power consumption, lower carbon emissions and environmental impacts) - a concern for the natural environment $[12,13]$.

We acknowledge that GIT/GIS initiatives and acting in environmentally friendly ways is already - or will very soon be - recognized by customers and society, resulting in a competitive advantage for organizations, i.e. by lowering costs and enabling a differentiation advantage [14]. Costumers increasingly differentiate between companies that "effectively contribute to sustainability and those that do not" [15]. Thus, GIT/GIS can be a key enabler and trigger for both sustainable business transformation [16] and, potentially, also of a world in which corporate success is measured in not only achieving economic, but also societal and environmental value [15].

We argue that, in recent times, in which digital transformation is seen as key for the wellbeing of global welfare [17], novel and resource-conserving GIT/GIS 
has the potential to both (reactively) address modern digitalization and environmental challenges, and to proactively create sustainable benefits [18-20].

However, we also see that society is yet to realize the full potential of GIT/GIS, to fully embrace and promote research into it [21]. This may also be because it is generally believed that interest in GIT/GIS is dwindling. While we agree that the initial hype around the topic has passed, overcoming this critical point may be particularly valuable for a technology or business application, since it is expected to be further processed with realistic expectations about outcomes [22].

Taking this as a starting point, we seek to better understand GIT/GIS adoption drivers, outcomes, and the forces that influence the implementation of green technologies in organizations [7, 11, 23, 24], contributing to both research and practice. As a theoretical perspective, we agree with other researchers (e.g. [19]) that, to fully pursue beneficial GIT/GIS initiatives, and make use of it as a contribution to both digitalization and societal changes, the topic needs to be put into a broader perspective. We also intend to answer calls for a theoretical framework to structure GIT/GIS research $[11,24]$. Traditional adoption frameworks are only partially suitable to frame GIT/GIS adoption, since they traditionally only focus on the individual and organizational levels. Thus, they neglect societal and governmental movements (e.g. the Paris Climate Agreement) that at some point will also affect business, but also altered environmental conditions (e.g. humandriven climate disruption [25]), as a driving force of sustainable technology adoption. Concerning practice, we acknowledge that a great many organizations are undertaking environmental efforts [11, 18, 20, 26], but that only a few (e.g. Tesla Motors) are committed to fully embracing and using green technology endeavors. Thus, we seek to support initial but also deepening organizational GIT/GIS endeavors by providing them with a full spectrum of relevant factors for GIT/GIS adoption.

A literature review approach has proven suitable for providing an overview and structuring insights into contemporary phenomena, such as GIT/GIS (e.g. [8, 11, $27,28])$. We summarize models and frameworks on GIT/GIS adoption and integrate them into a cognitive adoption framework. We include perspectives of the natural environment, society and individuals, since organizations "are not the only relevant actors in the global sustainability area" [15].

We will first present our framework of analysis before elaborating on our research method. We then present our literature review results by outlining the individual building blocks, which we then integrate into an integrative GIT/GIS adoption framework. We discuss the framework by highlighting further research implications, and close by pointing out limitations as well as theoretical and practical implications.

\section{Analysis framework}

We will now specify our framework of analysis as a basis for our to-be-developed GIT/GIS adoption framework, which is built on three assumptions: First, we regard organizational innovation adoption as a desirable process. It is initiated by reactive or proactive strategic decisions concerning internal or external drivers that activate and energize organizations with the potential for increased performance [29]. Although it may simplify the complex underlying processes, we decided to use driver to reflect proactive implications, and practical link of the term. Second, we regard GIT/GIS as a desirable capability with which to establish a competitive advantage to for instance pursue a differentiation strategy [14]. Third, and separating it from other innovations, GIT/GIS may be used to address ongoing global climate disruption, as one of society's severe challenges, by supporting the preservation of the natural environment [25]. Thus, GIT/GIS needs to be set in relationship to an $\mathrm{AE}$, to the natural environment, and to society [15].

We distinguish between two complementary dimensions: i) adoption phases and ii) adoption levels. In the first dimension, we conceptualize innovation diffusion as an iterative, three-stage process (preadoption, adoption, and post-adoption) that bridges an initial as-is state (outset) and a future to-be state (outcome). This is based on the conceptualization of innovation adoption as a sequential process through which an AE passes from getting knowledge about an innovation, forming a positive or negative attitude towards it, to making an adoption decision that is then reinforced by consecutive behaviors [9].

This process bridges an entity's current and a future state, which we further regard as two district states of for instance organizational resources, capabilities, and employee skills (RCS). We term the beginning or starting point the outset phase. It defines the initial RCS combination and is taken as a baseline to be compared to the outcome phase, as the projected or documented RCS state after adopting and implementing GIT/GIS.

In the second dimension, we model the natural environment, the societal level, the organizational level, and the individual level, for multiple reasons. First, integrating various ecological, economic, and social dimensions has become a practice. It is for instance termed the triple-bottom-line principle, and reflects that organizations need to adhere not only to economic goals (single bottom line), but should also emphasize social and ecological goals [21]. This also reflects the claim 
that IS research should change its primarily inward orientation to become more inclusive, by incorporating also social and environmental challenges [30, 31].

Second, AE GIT/GIS innovation actions may be caused by one or multiple factors, for instance an altered organizational culture [27], societal drivers (e.g. social and cultural influences [32], or legislative pressures [13]). Also, governments, media, and other institutions (e.g. NGOs) may influence an AE's actions, creating both opportunities and challenges [15].

Third, organizations are a central but "not the only relevant actors in the sustainability arena" [15]. For instance, individual persons also determine organizational actions and activities. Management lays out strategic and tactical courses of action (e.g. to adopt GIT) that are then operationalized and put into practice by staff. Thus, not only their actionable outcomes should be integrated, but also external factors (e.g. the perceived state of the natural environment) that determine these outcomes.

Fourth, and in contrast to traditional innovations, GIT/GIS adoption can have implications and outcomes for individuals, society, and especially the natural environment that first manifest in the long-term and medium-term $[14,33]$.

\section{Research method}

Our research is a literature review that has synthesized and integrated literature from IS journals and conferences. To get a comprehensive picture, we first only used only 'Green IT' and 'Green IS' as search terms for literature searches in AISeL and Business Source Premier Database in EBSCOhost. We chose these, since they cover almost the entire spectrum of conference and journal publications most relevant to the academic IS community.

We then did additional searches that combined these terms with 'adoption', 'innovation adoption', 'adoption
Table 1: Sample and construct overview

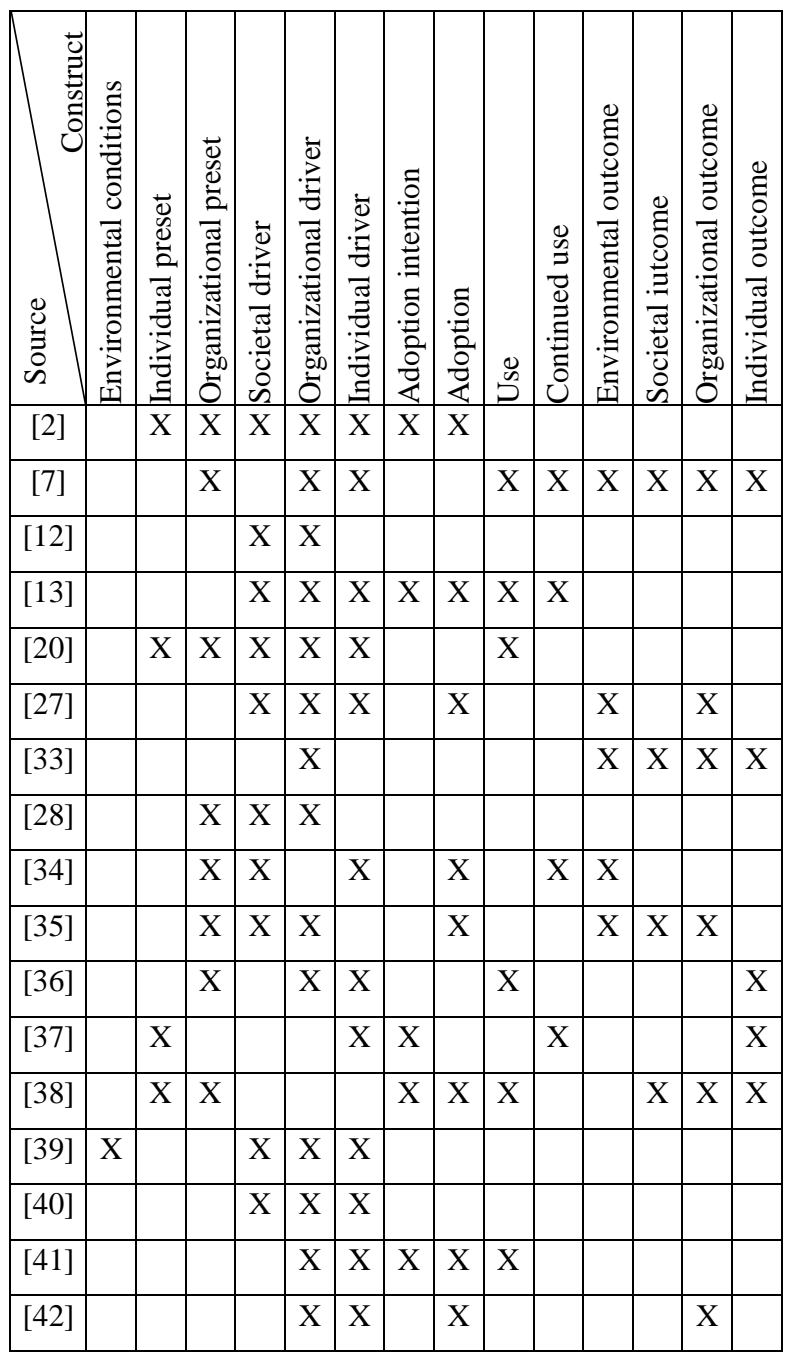

model', 'adoption framework', 'determinants', 'adoption outcome', and 'outcome'. To cover all the studies published since the origin year of the term GIT

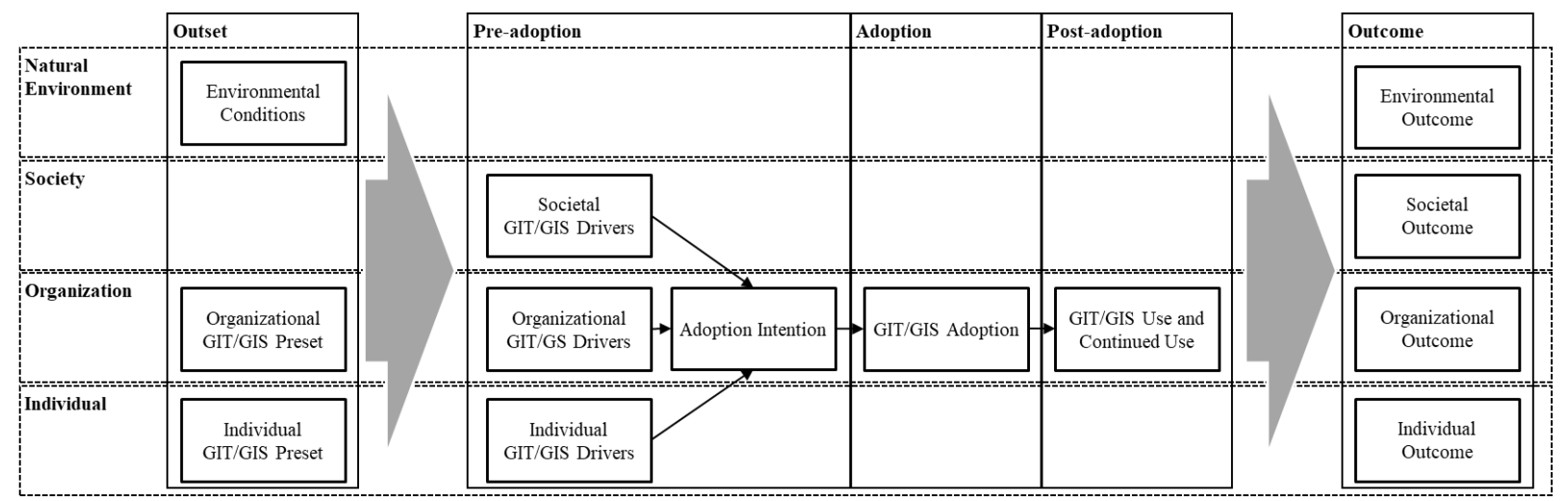

Figure 1. Integrative GIT/GIS adoption framework 
[5], we set 2007 as the only search date limit. The initial sample contain 203 papers. Similar to other research [5], and as andication of the position of GIT/GIS on the Gartner Hype Cycle [22], we also identified 2010 to 2014 as the years with the most publications about GIT/GIS. After eliminating duplicates and papers that, according to the abstract, did not fit our scope, the final sample contained 129 publications (98 conferences, 24 journals, 7 other). From these, we used 18 articles that concerned GIT/GIS adoption models, frameworks, determinants, and outcomes. Nine concerned organizational or individual readiness, 15 addressed environmental, societal, organizational, or individual drivers, and five addressed adoption intentions. 12 addressed adoption, use, or continued use, while nine concerned environmental, societal, organizational, or individual outcomes. Table 1 presents an overview.

\section{A GIT/GIS adoption framework}

As presented above, the identified GIT/GIS adoption models and frameworks have two dimensions: adoption phase (outset, pre-adoption, adoption, post-adoption, and outcome) and level (environmental, societal, organizational, and individual). During our literature review, we realized that the initial five-phase view must be specified by a distinction between the intention to adopt GIT/GIS, as part of the pre-adoption phase, and GIT/GIS use and continued use, as part of the postadoption phase. We integrated the identified GIT/GIS adoption criteria and outcomes on the outlined levels into a GIT/GIS adoption framework (as illustrated in Figure 1). However, it is to be understood as illustration of an overall cognitive process, rather than a specific innovation adoption process.

\subsection{Adoption phase: Outset}

Before addressing adoption, we must look at the context for GIT/GIS adoption, since these characteristics are linked to adoption [2]. We found that the outset phase reflects the natural environment as well as organizational and individual readiness (see Table 2).

4.1.1. Environmental conditions. We recognized that only selected contributions of the identified literature directly address and incorporate the natural environment, since they don't enact upon themselves, but are enacted through other drivers. The identified paradigmatic conditions stem from changing environmental conditions (e.g. global climate disruption), as well as pollution and the diminishing of rare and valuable resources [39].
4.1.2. Organizational preset. Organizational factors are closely linked to two theoretical constructs: i) the Technology-Organization-Environment (TOE) framework [2, 28, 34], and the GIT readiness [2, 20, 28, 35]. TOE is an organizational-level theory that considers the technological, organizational, and environmental contexts to be key for an organization's innovation adoption decision [43]. In this theory, technological context refers to the already available technology, IT, and their characteristics in the company $[2,34]$. We regard it as a key determinant of organizational GIT/GIS adoption, since GIT/GIS will primarily be adopted in organizations that have large IT assets or are undertaking green technology initiatives [2], since these technologies provide an ideal basis and platform for initial or further GIT/GIS initiatives. Organizational context describes various hard factors, such as structural aspects of branch, corporate citizenship, and company size, or other formalized structures that may enable internal innovation processes, such as communication structures [2, 34]. They also describe soft factors such as work standards, normatively acceptable behaviors, and organizational culture. Especially soft factors make a substantial difference to which of the four GIT/GIS adoption approaches (e.g. green-washing or the deep green approach) an organization intends to pursue [36]. Environmental context is the third pillar of the TOE framework and contains external influences, such as legislative and governmental regulations, as well as market structures and characteristics [2, 34].

GIT readiness captures internal factors of perceived i) organizational readiness, ii) institutional readiness,

Table 2. Outset phase factors

\begin{tabular}{|l|}
\hline Environmental conditions \\
- Environmental conditions \\
\hline Organizational preset \\
Technology factors \\
- Available and installed (information) technologies \\
Organizational factors \\
- Hard factors (e.g. corporate citizenship, company size, \\
policies, governance) \\
- Soft factors (e.g. work standards, practices, normatively \\
acceptable behavior, organizational culture and attitude) \\
Environmental factors \\
- National and international regulations \\
- Market structures and characteristics \\
- Stakeholder pressures \\
\hline Individual preset \\
- Attitude (e.g. intrinsic motivation, mindset, experience) \\
- Actions (e.g. manager leadership) \\
\hline
\end{tabular}


and external factors of iii) value network readiness. Organizational and institutional readiness describes five factors: attitude, policy, practice, technology, and governance [28] as well as ability [35], as a unique combination of adoption determinants. Further, the value network captures the readiness of a company's external stakeholders (e.g. suppliers, or customers). If determinants along the internal dimensions are perceived to be excessive, or if stakeholders regard GIT/GIS adoption negatively, these initiatives are unlikely to be initiated at all [2]. Since TOE is well accepted and theoretically founded, we take it as a basis for merging its components with GIT readiness.

4.1.3. Individual preset. At the individual level, we identified individual attitudes and actions (e.g. intrinsic motivation, green mindset, managers' leadership, and past experience) [37, 38]. Concerning the first two factors, users and managers engage more in GIT/GIS adoption if their green ambitions derive from pleasure and self-determination to the cause. Concerning the latter two factors, leaders not only serve as role models to other employees, but their own actions and initiatives also influence further actions.

\subsection{Adoption phase: Pre-adoption}

We found that both internal and external drivers influence organizational GIS/GIT adoption. While recognizing that the literature contains many specific categorizations of these drivers (e.g. cost reduction; demands from legal and regulatory requirements; sociocultural and political pressures; enlightened selfinterest; a collaborative business ecosystem; new market opportunities) [12], we decided to categorize these drivers into three abstract categories: regulatory, economic, and ethical drivers [2]. We understand regulatory drivers as actions initiated to meet voluntary or mandatory demands. Economic drivers refer to actions with efficiency improvement or cost reduction intentions, while ethical drivers refer to sustainable and normatively good behaviors that seek social, global, and local recognition. Depending on the perspective, these may be internal, external, or both to an organization.

Besides the organizational and individual levels, GIT/GIS intentions may also be initiated by societal concerns. We identified the drivers that directly originate from society or the general public, but also from public institutions, such as governments [20] or non-governmental organizations (NGOs) (e.g. Greenpeace) [35]. These directly or indirectly influence regulatory frameworks, which require or set incentives for adherence to green practices (see Table 3).

\section{Table 3: Drivers of adoption intentions}

\section{Societal drivers}

Regulatory societal drivers

- National and international legislative, regulatory, and compliance requirements

- Public or social demand for adherence to green practices

Economic societal drivers

- Financial incentives (e.g. avoid liability risks)

Ethical societal drivers

- Responsible business practices and corporate citizenship

- Normative pressures and cultural expectations

Organizational drivers

External organizational regulatory drivers

- Social, cultural, and political regulations

- Professional network, customer, and vendor requirements

- Equity holder norms and competitors

Internal organizational regulatory drivers

- Corporate citizenship; strategy practices and processes

- IT, GIT, and GIS governance and policies

- Knowledge and technological capabilities

- Internal stakeholder regulations (e.g. top management)

External organizational economic drivers

- National and international pro-environmental grants

- Industry, competitor, and vendor pressures

- Equity holder pressures and customer expectations

Internal organizational economic drivers

- Green strategy (e.g. use of renewable energies,)

- Efficiency incentives (e.g. hardware consolidation, virtualization, complexity reduction)

- Investment incentives (e.g. reduce lifecycle cost)

- Investment concerns (e.g. budget or capacity concerns)

External organizational ethical drivers

- Global and local community incentives

- NGO incentives

Internal organizational ethical drivers

- Corporate culture (e.g. shared vision)

- Business ethics (e.g. responsible business practices)

- Managerial attitudes (e.g. towards green practices)

Individual drivers

Individual regulatory drivers

- International and national policies

- Management policies and leadership

Individual economic drivers

- Intrinsic motivation (e.g. improve sustainability)

- Extrinsic motivation (e.g. financial incentives or fines)

- Attitudes and perceptions (e.g. perceived benefits)

Individual ethical drivers

- Attitude (e.g. identification with green practices)

- Actions and skills (e.g. top-down or bottom-up influence) 
4.2.1. Societal drivers. Regulatory societal drivers, initiated by national, international, and professional institutions can initiate, set up, and enforce coercive GIT/GIS pressures. These may deal with energy efficiency, waste and recycling policies, or other environmental protection principles [27, 28, 35].

We identified economic societal drivers as primarily tax, or fine driven influences, since governments raise fees or compensation for environmental pollution or environmentally related incidents and accidents [20].

Ethical societal drivers are usually initiated by environmental NGOs (e.g. Greenpeace) or institutions that seek to influence businesses, and whose actions generally receive much attention. Organizations for instance may shift towards more sustainable actions, if other companies, customers, or equity holders respond positively to these drivers. [20,35].

4.2.2. Organizational drivers. GIT/GIS adoption owing to coercive pressures, such as external organizational regulatory drivers, may arise from external stakeholders (e.g. investors, customers) who influence the organization's public image, or can also result in legal consequences concerning disobeying government laws $[2,35,39,40]$. Especially good reception of environmental actions by members of the own or a competing organization may lead an organization to take up or increase its GIT/GIS adoption initiatives [27]. Such initiatives can also be driven by the industry, since it can be in an organization's interest to establish and meet certain industrywide legal or de facto standards, in order to reduce corporate, financial, or customer risks [20].

Normative pressures associated with internal organizational regulatory drivers are also linked to the TOE framework. Institutionalized as actions that professionalize, standardize, or refocus an organization's environmental performance, or control, they target internal and external stakeholder requirements [7, 27, 39, 41]. Some of these factors, for instance top management or strategic influences, may also inhibit or prevent GIT/GIS adoption.

External organizational economic drivers are also primarily initiated by external stakeholders (e.g. investors or customers), since non-adherence to meeting demands may lead to significant economic losses [39, 40]. They may be also driven by an organization's intention to mimic its competitors, to promote its own business model, to reduce uncertainty for customers, or to limit exposure to similar external pressures [20]. It may also create an (initial) technical superiority, which can then lead to a relative advantage $[27,35,39]$.

Often, internal organizational economic drivers stem from the desire to reduce costs (e.g. power, cooling, or real estate), to increase IT efficiency, or to use IT to reduce costs (e.g. fleet management, dynamic vehicle routing) $[2,33,39]$. However, they may also be characteristic to an organization or its IT strategy [42]. We identified that technical compatibility determines initial and further GIT/GIS adoption, since significant technological or organizational changes may lead to staff resistance and thus unprofitable investments [27].

We found that external organizational ethical drivers can be caused by NGOs that consider the natural environment as normatively worth protecting [35] and thus seek to initiate organizational behaviors towards environmentally friendly practices [20].

Internal organizational ethical drivers urge organizations to link their "business to socially accepted norms of going green such as reducing emission, recycling, reuse and electronic waste management" [2]. They may institutionalize a supportive (e.g. sustainability driven) or an opposing corporate culture (e.g. purely profit-driven) [35, 39].

4.2.3. Individual drivers. Individual regulatory drivers can be understood as initiatives started by internal stakeholders (e.g. management) that have the regulatory force to alter an organization [20, 27, 40]. They set formal or informal norms, practices, and standards for behaviors that can drive an organization towards green readiness or improvement $[34,36]$.

Individual economic drivers may originate from corporate managers and their promotive or depressive motivation for green technologies' benefits. Although efficiency incentives are often only beneficial in the short term, they may also lead to GIT/GIS capabilities and improved long-term competitiveness [40-42]. Individual stakeholders (e.g. employees, investors) may also be motivated by own economic incentives (e.g. financial bonuses or fines) or may transfer personal experiences to their employing organization to translate individual benefits into corporate ones [20,37].

Individual ethical drivers stem from stakeholders (e.g. consumers, employees, managers) with an environmental mindset, sentiments, values, and norms $[27,36]$. These external and internal stakeholders may influence employees, who may then stimulate sustainability values within an organization [27] to initiate or extend its GIT/GIS endeavors [7] in order to improve its image [40]. However, opposing individual attitudes on for instance green technologies' usefulness may have a negative influence.

4.2.4. Adoption intentions. We identified only a few sample studies that stress the gap between awareness of the environment and environmental actions. Of these, one makes an argumentation distinction [2]. Another 
finds empirical evidence for differences between organizations that have i) not yet planned or implemented, ii) planned, iii) implemented, or iv) implemented and further plan to implement GIT [41]. A third study highlights the difference and stresses that intention to adopt GIT/GIS - not adoption - is influenced by technological attributes, the organization, and environmental factors [38].

At the individual level, attitude to GIT/GIS adoption may be positively or negatively influenced by external regulations [37].

\subsection{Adoption phase: Adoption}

Compared to intention to adopt GIT/GIS, GIT/GIS adoption deals with de facto implementation [2]. Some $[2,35,41]$ view this stage as the outcome of a GIT/GIS adoption or the start of a GIT/GIS maturation process [34]. Others link it to resource adoption and capability, and skill building, stressing it as a vehicle towards a competitive advantage (e.g. [27, 42]). Further, some (e.g. [38]), take a more diverse approach, regarding GIT/GIS adoption and implementation as a process with individual, organizational, and social outcomes. We follow the this view and add environmental outcomes.

\subsection{Adoption phase: Post-adoption}

We realized a necessity to distinguish between adoption, use, and continued use of GIT/GIS. Adopting a specific technology is not enough, but they need to be applied, since it is "not technologies per se, nor how they may be used in general that matter, but the specific technologies in practice" [7]. Also, ensuring that GIT/GIS is not initially, but continuously used ensures positive, long term outcomes. We came to realize that the terminology of use and continued use of GIT/GIS varies between the sample authors (e.g. "proenvironmental IT practices" [36], "green IT practices" [20], "Green IT in practice" [7], or "Green IT Maturation" [34]). However, most refer to a GIT adoption understanding of incorporating "ecological principles and energy-efficient operations into its technology life cycle" [20] in terms of design, production, purchase, utilization, and disposal.

Furthermore, only few sample studies have included additional GIS aspects of extensive success management [41] or individual moral and social beliefs [13]. Some researchers theorize that, after initial GIT/GIS adoption, organizations will adopt additional measures to further reduce their environmental impacts, which may exceed GIT/GIS initiatives (e.g. by planting trees) [34]. Thus, we emphasize that use and continued use of GIT/GIS in organizations (i.e. usage duration,
Table 4. GIT/GIS adoption outcomes

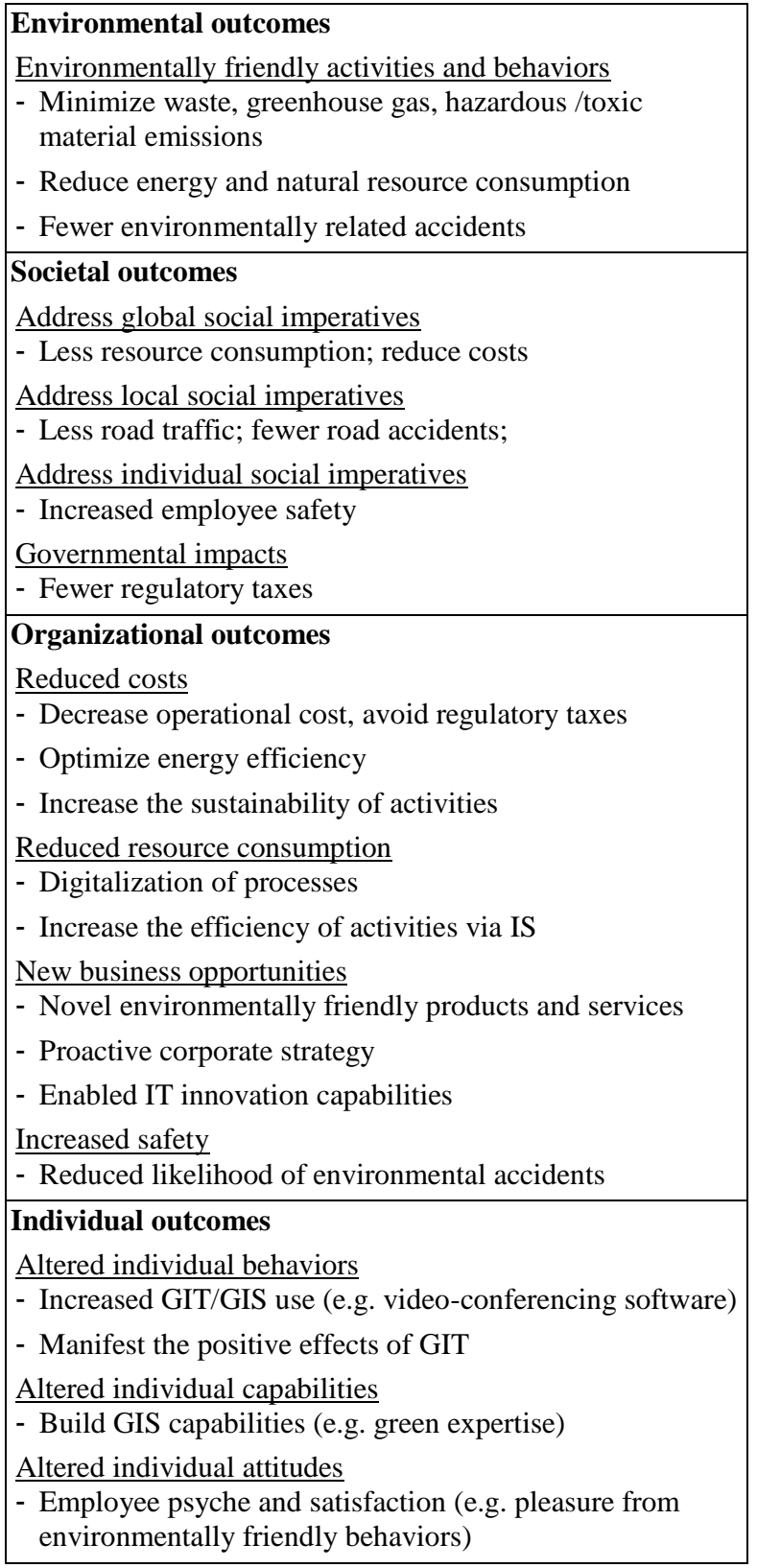

frequency, and intensity [13]), but also demonstrating the GIT/GIS benefits (e.g. cost savings through improved material utilization [7]), may cause a continuous use of GIT/GIS [37].

\subsection{Adoption phase: Outcome}

GIT/GIS adoption outcomes differ to those of other technologies. Besides organizational-level and individual-level outcomes, they incorporate societal and environmental outcomes $[33,38]$ (see Table 4). 
4.5.1. Environmental outcomes. We understand these as "benefits to the natural environment" [33]. GIT primarily affects the environment at the end of the pipe by reducing the impacts along a product's lifecycle that don't necessarily alter production processes [36]. Environmental GIS also focus on capability building, modifying (e.g. updating), optimizing (e.g. function enrichment), consolidating (e.g. server shutdown), or increased use (e.g. video-conferencing) of tangible and intangible IS [33]. Producing environmentally friendly (IT) products as a GIS outcome in the long term also affects the natural environment [7]. Organizations may exceed technological green endeavors, for instance by planting trees for further neutralization of emissions.

4.5.2. Societal outcomes. Society can benefit from GIT/GIS adoption from the production and use of environmentally sound products that meet predefined environmental regulations [7]. Also, IS usage can have societal crosslinked impacts, since computerized optimization of delivery routes can reduce traffic jams, road accidents, and vehicles' fuel consumption [33], yet reduce the amount of taxes and environmental fees [7].

4.5.3. Organizational outcomes. At the organizational level, GIT/GIS adoption outcomes are mostly measured concerning their ability to create a (sustainable) competitive advantage. We found that GIT and GIS may also be differentiated by their outcomes. GITs primarily focus on technology. Thus, GIT in practice (as sustainable and efficient computer resource uses) can for instance reduce costs and emissions and can minimize risks by avoiding penalties. Thus, GIT can create an initial and limited competitive advantage [7]. Since GIS also incorporates sustainable management activities and capability building [8], they focus on longterm ecological behaviors, and may thus create sustainable economic activities [27].

4.5.4. Individual outcomes. The individual level may be the most crucial level for both GIT/GIS adoption and use and for its continued use, since the decision to (not) adopt and use GIT/GIS is made by top management and the user level [7, 27]. It may for instance be evaluated concerning its criteria of technological complexity, ease-of-use, relative advantage [27], and learning, understanding, and incorporating GIT/GIS advantages for individual strategic value [33].

\section{Discussion and research implications}

The result of our literature review is an integrative framework that links individual, organizational, societal, and environmental GIT/GIS adoption drivers and outcomes. It integrates multiple studies that highlight individual perspectives on GIT and GIS that, for instance, either selectively present GIT adoption factors (e.g. [39, 40]), practices (e.g. [36]), or a highlevel overview of the GIT adoption process (e.g. [2]).

Our framework proposes an initial configuration of environmental conditions, and an organizational and individual preset. These factors determine societal, organizational, and individual factors that initiate an organization's intention to adopt, use, and continuously use GIT/GIS, which further lead to environmental, societal, organizational, and individual outcomes.

Based on these findings, we want to discuss some apparent perspectives and their implications for further research: First, our framework documents environmental conditions, as well as organizational, and individual factors, as outset factors of GIT/GIS adoption. Nonetheless, we theorize that certain societal conditions also influence GIT/GIS adoption. These may for instance be relatively stable cultural paradigms that manifest in a variety of cultural concerns and actions (e.g. environmentalism), but also fundamental economic and political assumptions (e.g. capitalism). Researching the interactions of these stable societal determinants with GIT/GIS may prove valuable for the further development of GIT/GIS adoption mechanisms, but also on how they may determine societal structures.

Second, our investigation revealed that incorporating the societal and environmental levels distinguish GIT/GIS innovations from traditional innovations. Accessibly, since GIT has been around for only a decade [5], considerably more emphasis has been put on researching it at the organizational and individual levels. We propose that uncovering societal and environmental impacts may be fruitful to establish novel categories for evaluating technologies, and to broaden the perspective. Technological impacts on these dimensions take longer to manifest and are therefore also harder to measure. However, in our view, these efforts are beneficial for long-term outcomes of GIT/GIS use, since these may underline GIT and GIS superiority over traditional IT and IS.

Third, we see that there are not only interactions but strong, interdependent ties between outcomes on the four levels. Digitalization of processes (organizational outcome) for instance translates to altered employee work procedures (individual outcome). Building GIS capabilities and behaviors (individual outcome) may also lower costs, since more sustainable resources and less energy consumption (organizational outcome) also translate into the generation of less taxes in resource and energy bills (societal outcome). These interactions should be further addressed, since they may enable policymakers to enforce policies that trigger the desired 
environmentally friendly outcomes and assess these policies' long-term implications.

This study has limitations. First, not all models and frameworks we used were empirically tested. While some have been tested in case studies [33, 35, 40] or surveys [7, 20], some are only conceptual [2, 28, 42]. Thus, the presented framework should be understood as a high-level overview that describes the overall context of GIT/GIS adoption between these two streams: the need for digital transformation and sustainable business. Further research may address this shortcoming by empirically validating our work while also addressing the general lack of empirical work on sustainability and GIT/GIS [20, 42]. Also, although we identified a multitude of both positive and negative factors, our work is not extensive, a constraint that further qualitative research may address.

Nonetheless, our work is beneficial for practice. Practitioners may take this work as a prompt to initialize first or advancing GIT/GIS endeavors in their organizations. As a starting point, an organization may choose to document employee attitudes and ideas (of the individual preset factors) to mobilize the employee base and initialize a transformation from within. It may also decide to take the identified outcome factors, to add to the factors a company reports on, to address its sustainability reputation. It may for instance choose to also report on the extent of reduced greenhouse gas emissions owing to the increased use of videoconferencing instead of employees taking business trips) or increased employee satisfaction (e.g. owing to home office work instead of commuting to work).

\section{Conclusion}

Organizations and society in general now face substantial challenges. On the one hand, digitalization requires businesses to further incorporate IT/IS assets to digitally transform their structures and processes. On the other hand, society - as customers, competitors, and vendors - is forced to increasingly behave in environmentally friendly ways to address the challenges of global climate disruption [25]. As assets that "minimize the negative environmental impacts of IS, business operations, and IS-enabled products and services" [8], GIT/GIS can help us to simultaneously pursue both goals.

We addressed the call for a framework to structure GIT/GIS research $[11,24]$, providing an overview that is detached from an organization's specific strategy and processes [14], and the shortcoming of present GIT/GIS research, which has focused on the organizational and the individual levels, by incorporating societal and environmental perspectives [11].
Based on the developed framework, we propose avenues for further research. In our view, especially the missing societal conditions of the outset phase, as well as the implications of the societal and environmental determinants and outcomes should be addressed.

\section{References}

[1] R. Nishant, T. Teo, and M. Goh, "Do Shareholders Value Green Information Technology Announcements?," Journal of the Association for Information Systems, vol. 18, 2017-08-31 2017.

[2] A. Molla, "GITAM: A Model for the Adoption of Green IT," in 19th Australasian Conference on Information Systems, Christchurch, 2008.

[3] M. T. Ijab, A. Molla, and V. A. Cooper, "A theory of practice-based analysis of Green Information Systems (Green IS) use," in 22nd Australasian Conference on Information Systems, Detroit, 2011.

[4] K. Erek, F. Loeser, and R. Zarnekow, "Reference Model for Sustainable Information Systems Management: Establishing a Holistic Research Agenda," 2012.

[5] D. Sedera, S. Lokuge, B. Tushi, and F. Tan, "Multidisciplinary Green IT Archival Analysis: A Pathway for Future Studies," Communications of the Association for Information Systems, vol. 41, no. 1, pp. 674-733, 2017.

[6] S. Brooks, X. Wang, and S. Sarker, "Unpacking green IT: A review of the existing literature," 2010.

[7] G. L. Lunardi, D. B. Dolci, A. C. Salles, and A. P. F. Alves, "Green IT: an Empirical Study regarding Organizational Actions and Impacts on Environmental Performance.," 2015.

[8] F. Loeser, "Green IT and Green IS: Definition of constructs and overview of current practices," presented at the Americas Conference on Information Systems, Chicago, Illinois, 2013.

[9] E. M. Rogers, Diffusion of innovations. Simon and Schuster, 2010.

[10] M. A. Hameed, S. Counsell, and S. Swift, "A conceptual model for the process of IT innovation adoption in organizations," (in en), Journal of Engineering and Technology Management, vol. 29, pp. 358-390, 7/2012 2012.

[11] G. L. Lunardi, A. P. F. Alves, and A. C. Salles, "Green IT Maturity: developing a framework based on practices and actions," in CONF-IRM, 2013.

[12] S. Murugesan, "Harnessing green IT: Principles and practices," IT professional, vol. 10, no. 1, 2008.

[13] C. Koo, N. Chung, and K. Nam, "Assessing the impact of intrinsic and extrinsic motivators on smart green IT device use: Reference group perspectives," International Journal of Information Management, vol. 35, no. 1, pp. 64-79, 2015.

[14] C. Henkel, A.-R. Seidler, J. J. Kranz, and M. Fiedler, "How to become a Sustainability Leader? The Role of IS 
Affordances in Enabling and Triggering Sustainability Transformations," 2017.

[15] T. Dyllick and K. Muff, "Clarifying the meaning of sustainable business: Introducing a typology from businessas-usual to true business sustainability," Organization \& Environment, vol. 29, no. 2, pp. 156-174, 2016.

[16] S. Seidel, J. Recker, and J. vom Brocke, "Sensemaking and Sustainable Practicing: Functional Affordances of Information Systems in Green Transformations," MIS Quarterly, vol. 37, pp. 1275-A10, 2013.

[17] OECD, "KEY ISSUES FOR DIGITAL TRANSFORMATION IN THE G20," OECD2017-01-12 2017, Available: https://www.oecd.org/g20/key-issues-fordigital-transformation-in-the-g20.pdf.

[18] D. Kiron and G. Unruh, "The Convergence of Digitalization and Sustainability," MIT SLOAN MANAGEMENT REVIEW, Whitepaper 2018.

[19] G. Unruh and D. Kiron, "Digital Transformation on Purpose," in Big Idea: Sustainability, ed, 2017.

[20] P. J.-H. Hu, H.-f. Hu, C.-P. Wei, and P.-F. Hsu, "Examining Firms' Green Information Technology Practices: A Hierarchical View of Key Drivers and Their Effects," Journal of Management Information Systems, vol. 33, no. 4, pp. 1149-1179, 2016.

[21] S. Seidel et al., "The Sustainability Imperative in Information Systems Research," CAIS, vol. 40, p. 3, 2017.

[22] Gartner. (2018, 05.06.2018). Gartner Hype Cycle. Available:

https://www.gartner.com/technology/research/methodologies /hype-cycle.jsp

[23] V. A. Cooper and A. Molla, "A Contextualist Analysis of Green IT Learning in Organisations," in International Conference on Information Resources Management, Vienna, 2012.

[24] B. Tushi, D. Sedera, and J. Recker, "Green IT segment analysis: an academic literature review," 2014.

[25] B. Tomlinson, Greening through IT: information technology for environmental sustainability. MIT Press, 2012.

[26] S. Brockhaus, S. E. Fawcett, A. M. Knemeyer, and A. M. Fawcett, "Motivations for environmental and social consciousness: Reevaluating the sustainability-based view," (in en), Journal of Cleaner Production, vol. 143, pp. 933-947, 2017.

[27] Q. Deng and S. Ji, "Organizational green IT adoption: concept and evidence," in 21st Americas Conference on Information Systems, Puerto Rico, 2015, vol. 7, no. 12, pp. 16737-16755.

[28] A. Molla et al., "E-readiness to G-readiness: Developing a green information technology readiness framework," in 19th Australasian Conference on Information Systems Christchurch, 2008.

[29] A. Subramanian and S. Nilakanta, "Organizational innovativeness: Exploring the relationship between organizational determinants of innovation, types of innovations, and measures of organizational performance," Omega, vol. 24, pp. 631-647, 1996.

[30] J. v. Brocke, R. T. Watson, C. Dwyer, S. Elliot, and N. Melville, "Green information systems: Directives for the IS discipline," 2012.

[31] R. T. Watson, M.-C. Boudreau, and A. J. Chen, "Information systems and environmentally sustainable development: energy informatics and new directions for the IS community," MIS Quarterly, pp. 23-38, 2010.

[32] S. Murugesan and G. R. Gangadharan, Harnessing green IT: Principles and practices, 2008. [Online]. Available.

[33] D. M. Simmonds and A. Bhattacherjee, "Smart Systems, Smarter Living: An Empirical Study of the Building Automation System in Organizations," 2015.

[34] R. Bose and X. Luo, "Integrative framework for assessing firms' potential to undertake Green IT initiatives via virtualization-A theoretical perspective," The Journal of Strategic Information Systems, vol. 20, no. 1, pp. 38-54, 2011.

[35] S. Karanasios, V. Cooper, H. Deng, A. Molla, and S. Pittayachawan, "Antecedents to greening data centres: A conceptual framework and exploratory case study," in $21 s t$ Australasian Conference on Information Systems (ACIS 2010), 2010.

[36] A. Molla, A. Abareshi, and V. Cooper, "Green IT beliefs and pro-environmental IT practices among IT professionals," Information Technology \& People, vol. 27, no. 2, pp. 129-154, 2014.

[37] C. Koo and N. Chung, "Examining the ecotechnological knowledge of Smart Green IT adoption behavior: A self-determination perspective," Technological forecasting and social change, vol. 88, pp. 140-155, 2014.

[38] X. Wang, S. Brooks, and S. Sarker, "Understanding Green IS Initiatives: A Multi-theoretical Framework," CAIS, vol. 37, p. 32, 2015.

[39] L.-D. Radu, "Determinants of Green ICT adoption in organizations: A theoretical perspective," Sustainability, vol. 8, no. 8, p. 731, 2016.

[40] S. Kurnia, M. M. Rahim, D. Samson, and S. Prakash, "Exploring the Adoption of Sustainable Supply Chain Practices in Australia: Current Practices and Adoption Motivations," in PACIS, 2014.

[41] N.-H. Schmidt, K. Erek, L. M. Kolbe, and R. Zarnekow, "Predictors of Green IT Adoption: Implications from an Empirical Investigation," in AMCIS, 2010.

[42] R. E. A. Rahim and A. Rahman, "Resource-based framework of green it capability toward firms' competitive advantage," in Proceedings of the 17th Pacific Asia Conference on Information System, Jeju Island, Korea, 2013, pp. 18-22.

[43] J. Baker, "The Technology-Organization-Environment Framework," in Information Systems Theory, vol. 28, Y. K. Dwivedi, M. R. Wade, and S. L. Schneberger, Eds. New York, NY: Springer New York, 2012, pp. 231-245. 